\title{
Efficacy of parasternal injection of bupivacaine on postopeartive pain for early extubation in patients undergoing coronary artery bypass surgery
}

\begin{abstract}
Objectives: The aim of this study was to determine the efficacy of parasternal injection of Bupivacaine on postoperative pain for early extubation in patients undergoing coronary artery bypass grafting.

Material and methods: Patients admitted throughout patient department for elective primary coronary artery bypass surgery into the surgical ward were enrolled for the study. A total of 35 patients were randomized into two groups, (Bupivacaine) group $113(37.14 \%)$ and (Placebo) group $215(42.85 \%)$. Patients were induced with standard anesthetic protocols. Bupivacaine injection $0.5 \%$ at $2 \mathrm{mg}$ per $\mathrm{kg}$ was diluted in $50 \mathrm{mls}$ syringe with normal saline and $50 \mathrm{mls}$ of normal saline was used as Placebo. Identical syringes marked with a study number of patient were delivered to the operating theaters. Both the surgeon and the anesthetist were blinded of the content of the solution. At the end of the procedure the injection was infiltrated into the parasternal wound before placing the sternal wires. Patients were assessed in the intensive care unit by the anesthetist for early extubation. At the time of extubation pain intensity was measured with Visual analogue scale system and recorded.
\end{abstract}

Results: A total of 35 patients were selected for the study.28 patients were able to complete the study as [Seven $(2.45 \%)$ out of 35 patients were excluded from the study secondary to prolonged ventilation 5(14.2\%), and reopening 2(5.71\%). Mean Visual analogue scale was $1.38 \pm 1.19$ and $6.13 \pm 2.92$ in the Bupivacaine and Placebo group respectively with a significant $(\mathrm{P}<0.001)$ requirement of rescue pain medication was nil in the Bupivacaine while $8(53.3 \%)$ patients were given rescue pain medication in the Placebo group. Mean difference of total ventilation time in the Bupivacaine and Placebo group was Bupivacaine - 5.8 \pm 1.3 and Placebo - $122 \pm 2.8$ hrs, which was statistically significant $(\mathrm{p}=0.002)$. Mean Partial oxygen pressure was found $208.23 \pm 42.57$ and $126.40 \pm 23.15$ in the Bupivacaine and Placebo groups respectively which was a significant value $(\mathrm{P}<0.001)$.

Conclusion: We conclude that parasternal wound infiltration of long acting local anesthetic Bupivacaine is an effective method of pain control after sternotomy. It facilitates early extubation with significant improvements in oxygenation and Visual Analogue Scale scales.

Keywords: postoperative pain, local injection of bupivacaine, analgesia, parasternal anesthetic, bupivacaine in cardiac surgery
Volume 6 Issue 4 - 2016

\author{
Salman Ur Rahman, Tariq Azam Siddiqi, \\ Akhtar Husain, Rehana Yaseen, Riffat Tanveer, \\ Saad Badar, Munir Afridi, Rafiq Khan, lqbal \\ Pathan
}

Department of Cardiac Surgery and Anaesthesia, National Institute of Cardiovascular Diseases Karachi, Saudi Arabia

Correspondence: Salman Ur Rahman, Department of Cardiac Surgery and Anaesthesia, National Institute of Cardiovascular Diseases Karachi, Saudi Arabia, Email drsalmankhan@gmail.com

Received: August 14,2016 | Published: September 01, 2016

\section{Introduction}

Pain is one of the major concerns of any surgical patient entering in the intensive care unit (ICU). In cardiac surgical patients, pain is mostly due to median sternotomy (or sometimes thoracotomy) and it seems to be higher on the first two days which is the usual length of stay at the cardiac ICU. ${ }^{1}$ Traditionally the pain after median sternotomy is treated best with parenteral opioid-based analgesics. Non-steroid antiinflammatory agents (NSAIDS) have failed to show significant benefit over opioids in cardiac surgical patients. However, opioid analgesics are well known for their major and sometimes dreadful side effects, especially on cardiac surgical patients who should be discharged from ICU on a fast tract protocol. ${ }^{1-5}$

Early extubation (fast track) has been the most significant advance in ventilatory care of the post open heart cardiac surgery patients. Over 30 studies $^{1,2}$ reported that early extubation (within eight hours) appears to be safe without an increased incidence of morbidity. A benefit of the practice may be cost savings associated with shorter intensive care unit and hospital length of stays. Early extubation is safe and improves cardiac function by increasing preload as capacitance blood volume shifts into the chest. To achieve this target of early extubation, the early postoperative period in cardiac surgical patients is the crucial time to establish stable haemodynamics, alert mental status and adequate pain control. ${ }^{5-9}$

Uncontrolled postoperative pain in cardiac surgical patients may contribute to cardiac morbidity through activation of the sympathetic nervous system, surgical stress response, and coagulation cascade. Increased sympathetic nervous system activity can increase myocardial oxygen demand by increasing heart rate, arterial blood pressure, and contractility.

Susan B et al., ${ }^{7}$ showed parasternal block with infiltration of local Bupivacaine in the sternotomy wound and mediastinal tube sites can be a useful analgesic adjunct in the first four to six hours after cardiac surgery. Panagiotakopoulos V showed that $64 \%$ of patients didn't experience any significant pain at all, 29\% experienced improved pain compared with the other cardiac surgical patients and only $7 \%$ of the patients enrolled in this study experienced the "usual" pain. ${ }^{6-24}$

Bar A and colleagues showed that patients who had a parasternal intercostal block with Ropivacaine had visual analogue and numerical 
rating pain scales approximately $50 \%$ less than those in the saline group at extubation; $(29.5$ standard deviation $(\mathrm{SD})=24.3 \mathrm{v} 53.2 \mathrm{SD}=$ 24.1, Ropivacaine versus saline, respectively, $\mathrm{p}<0.001){ }^{25}$

We sought to investigate the effect of parasternal block after sternotomy with local anaesthetic of $0.5 \%$ Bupivacaine infiltration. The rationale, therefore, was to achieve the goal of early extubation by reducing the postoperative pain with the help of local anesthetic drug. Thus reducing hemodynamic and pulmonary complications associated with prolonged ventilation, improve pain control, length of intensive care stay, hospital acquired infections and extra financial burdens.

\section{Material and methods}

Patients admitted through the outpatient department of National Institute of Cardiovascular Diseases from in the surgical ward for the first time for elective coronary artery by pass grafting and giving their consent, were enrolled for the study. They were randomized into two groups, group 1 (Bupivacaine group) and group 2 (Placebo group). The research officer and a staff nurse prepared coded solutions with the study drug or Placebo and were not directly involved in the clinical treatment of randomized patients. The solutions contained $2 \mathrm{mg} / \mathrm{kg}$ of Bupivacaine $(0.5 \%)$ with normal saline in a $50 \mathrm{mls}$ syringe (drug) and $50 \mathrm{mls}$ of normal saline in a $50 \mathrm{mls}$ syringe (Placebo) with no additives. Identical syringes marked with a study number of patient were delivered to the operating theaters. As the solutions were colourless and oduorless neither the surgeons, nor the anesthetists, scrub nurses and perfusionists knew the composition of the solution administered. The data collecting staff recorded the sample and theater number. Thirty five consecutive patients were randomly assigned to group 1 (Bupivacaine group) or group 2 (Placebo group). Only the operating cardiac surgeons with a ten years post fellowship experience were responsible for the infiltration of the injection into the parasternal spaces before the sternal closure with sternal wires. The mediastinal tubes were clamped, patient shifted to the intensive care unit and clamps removed. The anesthetist was responsible to assess and record the visual analogue scale.

This was a prospective, double blinded, randomized, Placebocontrolled study. It was performed at The National Institute of Cardiovascular Diseases with the approval of the local ethics committee of the NICVD.

The study was conducted over a six month period commencing from May 2009 to Nov 2009. Sample Size estimated with $95 \%$ of confidence interval and a power of $80 \%$ was $n=34$ with 17 in each group calculated with open-epi method. The patients of either gender, age range 30years to 70years and undergoing first time for isolated coronary artery bypass surgery were Included in the study.

We excluded the patients who did not give their consent as well as patients for congenital heart disease, poor left ventricular function (ejection fraction $<30$ ), pre-existing lung disease, duration of cardiopulmonary bypass ( $>120 \mathrm{~min})$, Pre-existing liver failure, known bleeding disorders, drug hypersensitivity, patients on more than one inotropic agent for haemodynamic support, patients on the Intra Aortic Balloon Pump, concomitant coronary artery bypass with valve procedures and patients for valve replacement.

\section{Operative technique}

Anaesthetic techniques and cardiopulmonary bypass procedure with a membrane oxygenator were standardized and maintained as follows:-
A. Pre-medication: Tablet DORMICUM $0.1 \mathrm{mg} / \mathrm{kg}, \quad P O, 2$ hours before surgery Induction: MORPHINE $0.15 \mathrm{mg} / \mathrm{kg}$ IVDORMICUM $0.02 \mathrm{mg} / \mathrm{kg}$ I PAVULON $0.1 \mathrm{mg} / \mathrm{kg}$ IV- STP $2 \mathrm{mg} / \mathrm{kg}$ IV- Infusion GTN in titrating doses if hypertension at induction.

B. Maintenance: PREBYPASS Oxygen, Nitrous oxide and Forane $0.5-3 \%$ in a titrating dose GTN infusion to control rise in BP.

C. On Bypass: MORPHINE $0.1 \mathrm{mg} / \mathrm{kg}$ at commencement of CPB. PAVULON $0.05 \mathrm{mg} / \mathrm{kg}$ at commencement of CPB MORPHINE $0.05 \mathrm{mg} / \mathrm{kg}$ at re warming temp of $35^{\circ} \mathrm{C}$.

D. Off Bypass: PAVULON $0.05 \mathrm{mg} / \mathrm{kg}$ at re- warming temp of $35^{\circ} \mathrm{C}$ FORANE $0.5-1 \%$ NO MORPHINE OR PAVULON AFTER THAT DOSE PROPOFOL $0.5-2 \mathrm{mg} / \mathrm{kg}$ when patient was in ICU. Any rise in $\mathrm{BP}$ to be controlled with PROPOFOL infusion.

At the conclusion of surgery, the patient was shifted into the intensive care unit. In the intensive care unit standard extubation protocols were observed to assess patients for extubation as follows:

Haemodynamic stability (systolic blood pressure $>90 \mathrm{mmHg}$ )

Satisfactory urine output $(0.5-1 \mathrm{ml} / \mathrm{kg})$

Normal arterial blood gasses (Ph-7.34 - 45)

Minimal chest drainage $(<3 \mathrm{ml} / \mathrm{kg}$ in the first three hours)

Spontaneous respiratory rate at $8-12$ breaths $/ \mathrm{min}$.

Amlseptable level of consciousness (obeys verbal command)

The time of extubation was documented and recorded. The intensity of pain after extubation was measured using the Visual Analogue Pain Scale in the intensive care unit by the anesthetist at extubation. The final outcome of the study was the mean pain scale on Visual Analogue Scale in Bupivacaine group less than the Placebo group at the time of extubation.

\section{Statistical analysis}

The data were analyzed on the (SPSS version 10.). Continuous response variables like $\mathrm{PaO} 2$, VAS and ventilation time were presented by Mean+/- SD; unpaired t-test was applied to compare the means between drug and Placebo groups. Categorical data like gender and requirement of analgesia were presented in terms of frequencies and percentages. Chi-square test or Fisher's Exact test were used to compare between the Bupivacaine and Placebo groups. Statistical significance was defined as $\mathrm{P} \leq 0.05$.

\section{Result}

From May 2009 to September 200935 patients were enrolled and randomized for the study. Following the inclusion criteria only 28 patients were able to complete the study. Seven $(20 \%)$ patients were unable to complete the study because of prolonged ventilation. $5(14.29 \%)$ patients had unstable hemodynamics and $2(5.71 \%)$ for reopening secondary to bleeding. Of the 28 patients $13(37.14 \%)$ were from Bupivacaine and 15(42.8\%) from Placebo group. There were 8 (61.5) males and 5 (38.5) females in the Bupivacaine group whereas 9 (60.0) males and 6 (40.0) females in the Placebo group ( $\mathrm{P}=0.934)$. The Visual Analogue scale remained (0-3) mean (1.38 \pm 1.19$)$ in the Bupivacaine group and non required rescue pain medication. While a mean of $(6.13 \pm 2.92)$ in the Placebo group. The values were significant for the difference in the mean Visual Analogue scale between the two groups $(\mathrm{P}=0.001)$ rescue pain medication was required in $8(53.3 \%)$ whereas none of the patients fron the Bupivacaine group required 
rescue pain medication. These values again reflected a significant difference between the two groups $(\mathrm{P}=0.002)$.

The partial oxygen pressure shows a trend of remaining higher in the drug group with a mean of $(208.23 \pm 42.57)$ in the Placebo group it remained at a lower scale with a mean $(126.40 \pm 23.15)$ these results reflect the respiratory comfort and ease of vital capacitythe difference was statistically significant $(\mathrm{P}<0.001)$.

Mean mechanical ventilation time was $5.8 \mathrm{hrs}$ in the Bupivacaine (CI 95\%-STD 1.28) and 7.8hrs in the Placebo group (CI95\%-STD 2.83 ) which was statistically significant $(\mathrm{P}=0.002)$ (Table 1).

Table I Describes the visual analogue scale for pain $\mathrm{PaO}_{2}$ (partial pressure of oxygen in $\mathrm{mmHg}$.Analgesia whether used or not represented as "yes" or"no"

\begin{tabular}{llll}
\hline Variables & $\begin{array}{l}\text { Groups } \\
\text { Bupivacaine } \\
(\mathbf{n}=1 \mathbf{3})\end{array}$ & $\begin{array}{l}\text { Placebo } \\
(\mathbf{n}=15)\end{array}$ & P values \\
\hline Gender & & & \\
Male & $8(61.5)$ & $9(60.0)$ & 0.934 \\
Female & $5(38.5)$ & $6(40.0)$ & \\
$\mathrm{PaO} 2(\mathrm{mmHg})^{\wedge}$ & $208.23 \pm 42.57^{*}$ & $126.40 \pm 23.15$ & $<0.001$ \\
Ventilation time (hours) & $4.92 \pm 1.26$ & $7.80 \pm 2.83^{*}$ & 0.002 \\
Visual analogue scale (VAS) & $1.38 \pm 1.19$ & $6.13 \pm 2.92^{*}$ & $<0.001$ \\
Requirement of analgesia & & & \\
Yes & $0(13)$ & $8(53.3)^{*}$ & 0.002 \\
No & $13(100)$ & $7(46.7)$ & \\
\hline
\end{tabular}

${ }^{\wedge} \mathrm{PaO}_{2}=$ Partial pressure of Oxygen

*Shows significantly higher value at $5 \%$ level of significance.

Use of rescue pain medication varied in both the groups. The Bupicacaine group had nil requirement of the rescue pain medication while $8(53.3 \%)$ were given opioids or analgesics for the in tolerable pain.

\section{Discussion}

There are numerous definitions of pain. The American Academy of Pain Medicine defines pain as - "An unpleasant sensation and emotional response to that sensation".

Pain is an objective parameter related to the degree of patient's tolerance. This is different from patient to patient. Poor or Uncontrolled postoperative pain in patients undergoing cardiac surgical procedures with either thoracotomy or sternotomy may contribute to cardiac morbidity through activation of the sympathetic nervous system, surgical stress response, and coagulation cascade. Increased sympathetic nervous system activity can increase myocardial oxygen demand by increasing heart rate, arterial blood pressure, and contractility.

There are numerous methods to assess the intensity of pain, usually measured in numbers or local phrases. Commonly methods used as scales like Verbal Rating Scale (VRSs), numerical rating scales (NRSs) and visual analogue scales (VASs). In Pakistan the Pakistan Coin Pain Scale (PCPS) is used commonly. ${ }^{2,3}$ We adopted for the VAS (visual analogue scale) which was found comparatively easier for our patient population. In addition to the pain intensity we also questioned the patients as to how much the patient is tolerating the pain. This not only helped us in categorization of the pain scale but also tailored the decision making in the use of rescue pain medication. Patient's awareness at the preoperative period about the procedure and feelings at the time of awakening on ventilator is an aide to alleviate the anxiety and a useful known method of controlling post operative pain.
Early tracheal extubation is a desirable goal for cardiac surgical patients and may lead to better respiratory function, decreased symptoms of depression, decreased cost and length of ICU stay. Over thirty studies ${ }^{25}$ have shown that early extubation (within eight hours) is cardiac surgical patients is beneficial without an increased incidence of complications. It is believed that early extubation is an integral part of the Coronary Artery Bypass Grafting fast-track recovery process. ${ }^{7}$

Cheng et al. ${ }^{20}$ have shown several potential benefits to early extubation. Early endotracheal tube removal triggers rapid return of ciliary function and improves respiratory dynamics and coughing. In fact, it has been proposed that early extubation should decrease the incidence of nosocomial pneumonias. The intrapulmonary shunt fraction improved significantly among patients extubated early. ${ }^{7,13,14}$ Moreover, mechanical ventilation itself can impair venous return and decrease cardiac output, thus prolonging Intensive Care Unit stay for adjustment of these parameters.

Tachycardia and hypertension are other responses that can omlsur secondary to mechanical ventilation and increase Intensive Care Unit by necessitating increased patient sedation. This has been an observation that patient and family satisfaction improve dramatically when patients are extubated early. ${ }^{21-23}$ This goal may be achieved after a good post operative analgesia.

Use of local anaesthetic agent in parastenal wounds has been shown to be a good adjuvant in pain control and a facilitator of early extubation. Susan B et al., ${ }^{7}$ showed parasternal block and infiltration of local Bupivacaine in the sternotomy wound and mediastinal tube sites can be a useful analgesic adjunct in the first four to six hours after cardiac surgery. Panagiotakopoulos V showed that $64 \%$ of patients didn't experience any significant pain at all, 29\% experienced improved pain compared with the other cardiac surgical patients and only $7 \%$ of the patients enrolled in this study experienced the "usual" pain. ${ }^{6,19,21-26}$

This randomized double blinded prospective study was based on White $\mathrm{PF}^{1}$ with a sample size of 35 patients for effect of Bupivacaine on pain for early extubation. Upon fulfillment of the extubation criteria in the intensive care unit the anesthetist performed tracheal extubation. The time of extubation and the pain scale were measured.

All of the patients in the Bupivacaine group remained within the (0-3) "no pain" or "mild pain scales", thus requiring no rescue pain medication or analgesia at extubation. The effect of parasternal block was observed from 6 - 8hours (mean 7.56hours), whereas in the Placebo group majority of the patients required rescue pain medication with either opioids or NSAIDs. This is in contrast to Wheatley $\mathrm{GH}^{5}$ where a lower dosage for opioids was used in the local anesthesia group compared to the Placebo.

Following injection of Bupivacaine for caudal, epidural, or peripheral nerve block in man, peak levels of Bupivacaine in the blood are reached in 30 to 45 minutes, followed by a gradual decline to insignificant levels during the next 3 to 6hours. The half-life of Bupivacaine is up to 8 hours $^{27}$ and the elimination half life is 4-6hours. Half life of Bupivacaine is increased in the elderly people. The technique of application of the injection should warrant the safety of bilateral internal thoracic vessels to avoid any untoward systemic effects. This may be achieved by putting the injections nearer to the medial sterna borders to avoid intravascular surge.

The primary end point in our study was pain intensity at tracheal extubation within eight hours (early extubation). We recorded the 
Visual analogue scale results of only those patients who were extubated within eight hours after surgery. Thus the rescue pain medication if, required would have been given at the time of extubation. As the pain scales and patient's comfort were both in mild tolerable states the rescue pain medication requirement was not justified. There were no complications as to the extubation were observed in the study population. Post operative course was smooth and the intensive care unit stay was unremarkable.

Prolonged mechanical ventilation was another hazard for the patients undergoing open heart surgery. Complications associated with mechanical ventilation may be avoided by weaning off the patients within six to eight hours as has been shown in several trials. The concept of early extubation following CABG was introduced in the 1970s. It did not gain widespread acceptance until recent years. Today the economical strategies have prompted universal interest in early extubation following open heart surgery. This strategy has been applied to both young and elderly patients. Low costs and savings have been demonstrated in younger, low-risk patient populations. ${ }^{25}$

In our study population the mean ventilation time in the Placebo group was $7.80 \pm 2.83$ hours. Out of 15 patients from the Placebo, $8(53.3 \%)$ patients required rescue pain medication, whereas in the Bupivacaine group the mean ventilation time was $4.92 \pm 1.26$ hours. This time period did not exceed more than six hours (mean-5.8hrs) in the drug group which is well within the time corresponding to the half life of the drug. This justifies the nil to mild pain scales and no rescue pain medication for the Bupivacaine group in our study. Moreover the application of the injection into the parasternal areas was carried out by experienced surgeons which may be an additional factor for a proper analgesic effect to be obtained.

Second most important factor in determining early extubation criteria was the respiratory component. Jc Ballantyne has shown that local anesthetics increased $\mathrm{PaO}_{2}$ (difference $4.56 \mathrm{~mm} \mathrm{Hg}, 95 \%$ CI 0.058-9.075) and decreased the incidence of pulmonary infections (RR $0.36,95 \%$ CI $0.21-0.65$ ) and pulmonary complications overall (RR 0.58 , 95\% CI 0.42-0.80) compared with systemic opioids. ${ }^{9}$ Another interesting finding in tis study that we found was a trend towards improved oxygenation in the Bupivacaine group as compared to the Placebo. This trend led us to assess the effect of the parasternal block on partial oxygen pressure in both the groups as well. Patients who had parasternal blocks did have better oxygenation at the time of tracheal extubation, a trend that continued more than 8hours period. The mean partial oxygen pressure was $208.23 \pm 42.57 \mathrm{mmHg}$ in the Bupivacaine group while in the Placebo it was $126.40 \pm 23.15 \mathrm{mmHg}$. these results when analyzed were statistically significant $(\mathrm{p}<0.001)$.

This may suggest a reduction in splinting with tidal-volume breaths and, therefore, atelectasis. However, bedside spirometry values were not considered. Although local anesthetic was not infiltrated around the mediastinal tube sites, still intrathoracic irritation was covered well. These tubes may have been the source of discomfort with the deep inspiratory maneuver and might be one of the reasons why tidal volumes were not improved in the non treatment (Placebo group). Even with this limited sample size, statistical significance was reached for our primary endpoint of early extubation (with in 8hours) with additional better oxygenation and improved Visual Analogue scales.

The Cochrane review on early extubation mentions that there is no evidence of a difference in mortality and morbidity rates between the study groups. But the results were consistent with the fact that early extubation reduces intensive care unit and hospital length of stay. ${ }^{7}$ A good pain control after sternotomy therefore, escalates vital capacity, improves oxygenation, prevents atelectasis, reduces incidence of post operative pneumonia, and reduces hospital stay. We recommend regarding the safety of this technique the dependence of a meticulous application of the infiltrate. Complications, although rare, include pneumothorax and hemothorax or bleeding from the site of injection. The local block should be performed under direct visualization before sternal wire closure by an experienced surgeon. The time before sternal wire closure also allows the surgeon to inspect the area for bleeding before chest closure. Care must be taken not to place the needle too lateral from the sternal border. Moreover negative aspiration during the intercostal block injections may inadvertently cause an intravascular injection.

\section{Limitations}

Small sample size was a big limitation in this study. We only included coronary artery bypass grafting patients, whereas emergency, valvular and congenital heart procedures should also be included which may widen the spectrum of the study. Another limitation was the systemic effects of the injection which could not be assessed. Larger trials with inclusion of all the categories of open heart surgery and variables like partial pressure of oxygen, carbon di oxide and there mean differences warrant further improved results.

\section{Conclusion}

We conclude that the parasternal infiltration of local anaesthetic Bupivacaine is an effective method of controlling post operative pain and facilitating early extubation. It has also shown improvements in arterial oxygenation and a significantly lower pain threshold after cardiac surgery.

\section{Acknowledgments}

None.

\section{Conflicts of interest}

Author declares there are no conflicts of interest.

\section{Funding}

None.

\section{References}

1. White PF, Rawal S, Latham P, et al. Use of a continuous local anesthetic infusion for pain management after median sternotomy. Anesth. 2003;99(4):918-923.

2. Priestley MC, Cope L, Halliwell R, et al. Thoracic epidural anesthesia for cardiac surgery: the effects on tracheal intubation time and length of hospital stay. Anesth Analg. 2002;94(2):275-282.

3. Merskey H. Classification of chronic pain: description of chronic pain syndromes and definitions of pain terms. Monograph for the subcommittee on Taxonomy International Association for the study of pam. Pain Suppl 3, Elsevier Science, Amsterdam. 1986.

4. Dowling R, Thielmeier K, Ghaly A, et al. Improved pain control after cardiac surgery: results of a randomized, double-blind, clinical trial. $J$ Thorac Cardiovasc Surg. 2003;126(5):1271-1278.

5. Roediger L, Larbuisson R, Lamy M. New approaches and old controversies to postoperative pain control following cardiac surgery. Eur J Anaesthesiol . 2006;23(7):539-550.

6. Wheatley $\mathrm{GH}$, Rosenbaum $\mathrm{DH}$, Paul $\mathrm{MC}$, et al. Improved pain management outcomes with continuous infusion of a local anesthetic after thoracotomy. J Thorac Cardiovasc Surg. 2005;130:464-468. 
7. McDonald SB, Jacobsohn E, Kopacz DJ, et al. Parasternal block and local anesthetic infiltration with levoBupivacaine after cardiac surgery with desflurane: the effect on postoperative pain, pulmonary function, and tracheal extubation time. Anesth Analg . 2005;100(1):25-32.

8. Hawkes CA, Dhileepan S, Foxcroft D, et al. Early extubation for adult cardiac surgical patients Cochrane Database Syst Rev. 2003;(4):CD003587.

9. Cheema S, Richardson J, McGurgan P. Factors affecting the spread of Bupivacaine in the adult thoracic paravertebral space. Anaesthesia. 2003;58(7):684-687.

10. Ballantyne JC, Carr DB, deFerranti S. The comparative effects of postoperative analgesic therapies on pulmonary outcome: cumulative meta-analyses of randomized, controlled trials. Anesth Analg. 1998;86(3):598-612.

11. Canto M, Sanchez MJ, Casas MA, et al. Bilateral paravertebral blockade for conventional cardiac surgery. Anaesthesia . 2003;58(4):365-370.

12. Deneuville M, Bisserier A, Regnard JF, et al. Continuous intercostals analgesia with $0.5 \%$ Bupivacaine after thoracotomy: a randomized study. Ann Thorac Surg. 1993;55(2): 381-385.

13. Sabanathan S, Smith PM, Pradhan GN, Hashimi H, Eng M, et al. (1988) Continuous intercostals nerve block for pain relief after thoracotomy. Ann Thorac Surg 46(4):425-426.

14. Joachimsson PO, Nyström SO, Tydén H. Early extubation after coronary artery surgery in efficiently rewarmed patients: a postoperative comparison of opioid anesthesia versus inhalational anesthesia and thoracic epidural analgesia. J Cardiothorac Anesth. 1989;3(4):444-454.

15. Gall SA, Olsen CO, Reves JG, et al. Beneficial effects of end tracheal extubation on ventricular performance. Implications for early extubation after cardiac operations. JThorac Cardiovasc Surg . 1988;95(5):819-827.

16. Kirklin JW, Barratt-Boyes Post operative care. (2nd edn), Cardiac Surgery, Churchill Livingstone, USA. p.196-221.

17. Kirvela O, Antila H. Thoracic paravertebral block in chronic postoperative pain. Reg Anesth. 1992;17(6):348-350.
18. Meissner A, Rolf N, Van Aken H. Thoracic epidural anesthesia and the patient with heart disease: benefits, risks, and controversies. Anesth Analg. 1997; 85(3):517-528.

19. Warltier DC, Pagel PS, Kersten JR. Approaches to the prevention of perioperative myocardial ischemia. Anesthesiology. 2000;92(1):253-259.

20. Panagiotakopoulos V, Theakos N, Charitos C, et al. Use of continuous subcutaneous anesthetic infusion in cardiac surgical patients after median sternotomy. J Cardiothoracic Surg. 2008;3:2.

21. Cheng DC. Fast track cardiac surgery pathways: early extubation, process of care, and cost containment. Anesthesiology. 1998;88(6):1429-1433.

22. Reis J, Mota JC, Ponce P, et al. Early extubation does not increase complication rates after coronary artery bypass graft surgery with cardiopulmonary bypass. Eur $J$ of Cardio-Thoracic Surg. 2002;21(6):1026-1030.

23. Nygard E, Kofoed KF, Holm S, et al. Effects of high thoracic epidural analgesia on myocardial blood flow in patients with ischemic heart disease. Circulation . 2005;111(17):2165-2170.

24. Salenger R, Gammie J, Vander Salm T. Postoperative care of cardiac surgical patients. Cardiac surgery in adults. In: Cohn LH, Edmunds LH, (Eds.), Cardiac surgery in the adult. McGraw-Hill, New York, USA. 2003. p.439-469.

25. Konstantakos AK, Lee JH. Optimizing timing of early extubation in coronary artery bypass surgery patients. Ann Thorac Surg. 2000;69(6):1842-1845.

26. Barr AM, Tutungi E, Almeida AA. Parasternal intercostal block with ropivacaine for pain management after cardiac surgery: a doubleblind, randomized, controlled trial. J Cardiothorac Vasc Anesth. 2007;21(4):547-553.

27. Miller RD, Cumlshiara RF, Miller ED. "Anesthesia" (5th edn). Churchil Livingstone, Philadelphia, Pennsylvania, USA. 2000. 